\title{
Méthodes numériques et simulations
}

\author{
G.J. Kearley et B. Nicolaï* \\ IRI - TU Delft, Mekelweg 15, 2629 JB Delft, The Netherlands \\ * Institut Laue-Langevin, 6 rue Jules Horowitz, BP. 156, 38042 Grenoble cedex 9, France
}

\begin{abstract}
Résumé. Ce cours introduit les principes fondamentaux des méthodes numériques et de simulation appliquées à la diffusion quasiélastique incohérente de neutrons. Les principales méthodes ab-initio ou semi-empiriques sont exposées brièvement. Leurs avantages et leurs inconvénients respectifs sont discutés. Nous exposons ensuite la méthode générale utilisée dans les simulations de dynamique moléculaire. Enfin, nous montrons comment on peut en déduire les grandeurs caractéristiques mesurables en diffusion neutronique quasiélastique ou inélastique.
\end{abstract}

\section{DESCRIPTION DES POSITIONS ATOMIQUES}

Dans le cas idéal, les positions atomiques sont connues grâce à la cristallographie, avec une précision généralement meilleure que le dixième d'angström. Elles sont le plus souvent exprimées en coordonnées cartésiennes, dans un système d'axes lié à la molécule qui cö̈ncide généralement avec celui de ses axes principaux d'inertie. Les programmes de simulation utilisent malheureusement plusieurs conventions pour décrire ces positions atomiques (ex : Shelx, pdb, etc...), mais des logiciels utilitaires permettent assez aisément de passer d'un format à un autre (ex : Babel).

Pour décrire une molécule, il est souvent plus pratique d'utiliser une matrice, connue sous le nom de matrice-z, qui contient toutes les informations géométriques sur la molécule, telles les longueurs de liaisons et les angles entre ces liaisons. On dit alors que l'on travaille en coordonnées internes. Cette convention est souvent préférée dans la mesure où ces coordonnées sont très similaires entre deux molécules voisines. La figure 1 montre une molécule formée de quatre atomes, numérotés de 1 à 4 . La description de sa structure nécessite de préciser les valeurs des trois longueurs de liaisons 1-2 (R1), 2-3 (R2) et 3-4 (R1), les deux angles B1 et B2 respectivement entre les liaisons 1-2 et 2-3 d'une part, 2-3 et 3-4 d'autre part ainsi que l'angle de torsion $\mathrm{T} 1$ entre le plan défini par les atomes 1,2, 3 et celui défini par les atomes $2,3,4$.

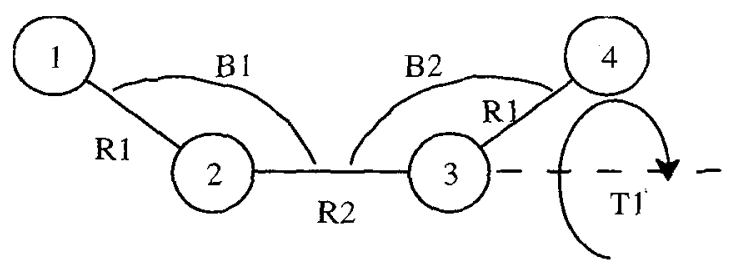

Figure 1: Description d'une molécule en termes de coordonnées internes.

Ces informations géométriques, exprimées en angströms et en degrés, seront regroupées sous la forme du tableau suivant : 
Tableau 1: Description de la molécule de la figure 1 en termes de coordonnées internes.

\begin{tabular}{|lllll|}
\hline 1 & & & & \\
1 & 2 & $\mathrm{R} 1$ & & \\
3 & 4 & $\mathrm{R} 1$ & & \\
2 & 3 & $\mathrm{R} 2$ & & \\
2 & 3 & $\mathrm{~B} 1$ & & \\
2 & 3 & 4 & $\mathrm{~B} 1$ & \\
1 & 2 & 3 & 4 & $\mathrm{~T} 1$ \\
\hline
\end{tabular}

où $\quad \begin{aligned} & \mathrm{R} 1=1,0 \\ & \mathrm{R} 2=1,5 \\ & \mathrm{~B} 1=120.0 \\ & \mathrm{~T} 1=45\end{aligned}$

Au cours d'une minimisation (voir paragraphe 3.1 plus loin dans ce cours), ces données géométriques (distances, angles ou torsions) seront considérées comme variables. L'avantage de ce formalisme est qu'il est possible de faire intervenir certaines variables plusieurs fois dans la matrice et de contraindre ainsi certaines distances ou certains angles à rester égaux au cours de la procédure de calcul. Par exemple, un rotateur méthyle $\mathrm{CH}_{3}$ sera considéré comme symétrique et les trois longueurs de liaison $\mathrm{C}-\mathrm{H}$ seront maintenues égales. Cette approximation permet de réduire le nombre de paramètres ajustables et par conséquent de diminuer le temps de calcul.

\section{L'ENERGIE DU SYSTEME}

Obtenir la description géométrique d'un modèle est généralement facile. Le travail véritable commence lorsqu'on désire décrire l'énergie du modèle. Plusieurs méthodes existent pour cela. Le choix de la méthode dépend de la précision, de la vitesse et de la quantité de calculs à effectuer. Leurs avantages et inconvénients respectifs sont comparés dans la figure 2.

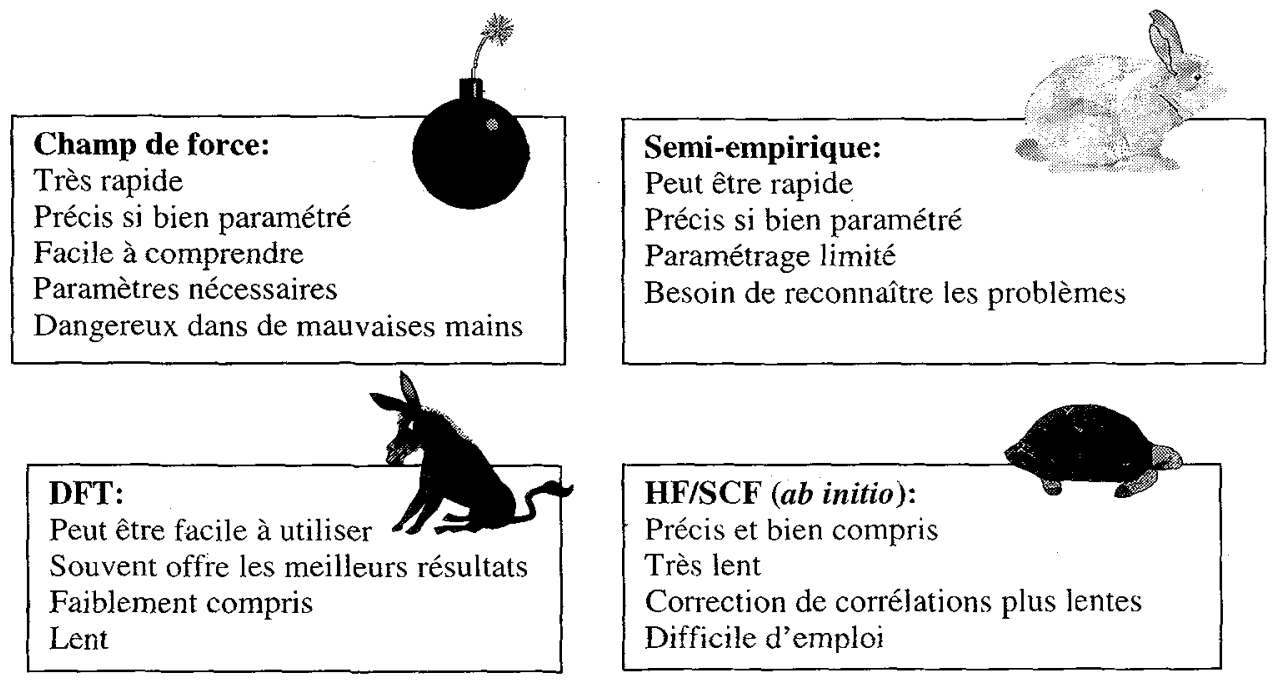

Figure 2 : Comparaison des différentes méthodes destinées à évaluer l'énergie d'un système. 


\subsection{Les méthodes empiriques ou de champ de force}

Les approximations principales de ces méthodes sont les approximations de Born-Oppenheimer et la possibilité de transférer des paramètres d'une molécule à l'autre.

L'énergie est principalement la somme de 3 contributions: une contribution interne, un terme de Van der Waals et une interaction de Coulomb. L'énergie interne fait intervenir la longueur des liaisons chimiques, les angles de déformation et les angles de torsion. Chaque contribution est paramétrée de la manière indiquée sur la figure 3 .
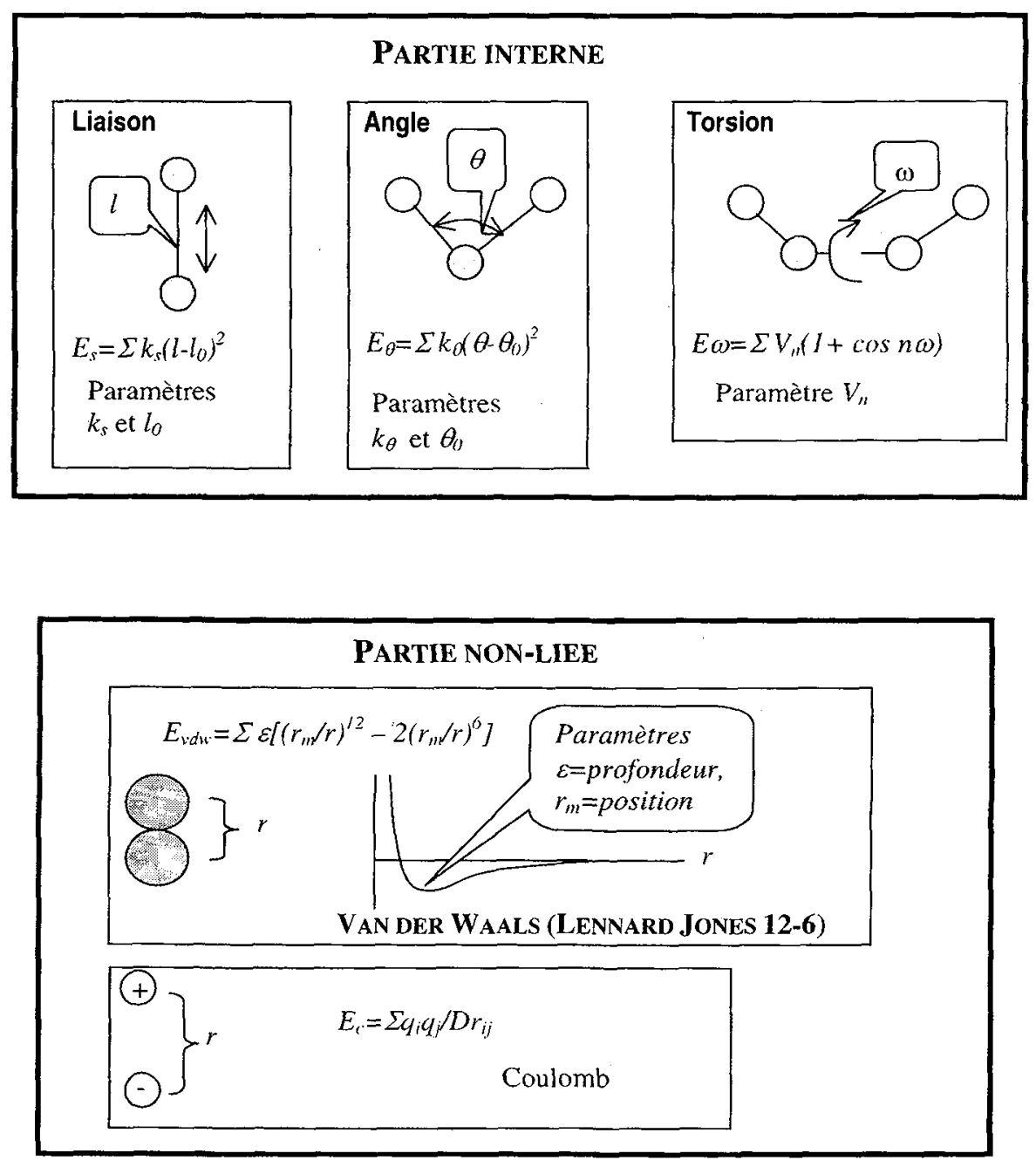

Figure 3 : Paramétrage des interactions internes et non-liées intervenant dans le calcul de l'énergie 
Les énergies liées aux élongations des liaisons chimiques et aux déformations des angles entre deux liaisons sont prises de la forme

$$
\begin{aligned}
& E_{s}=\sum k_{s}\left(l-l_{0}\right)^{2} \\
& E_{\theta}=\sum k_{\theta}\left(\theta-\theta_{0}\right)^{2}
\end{aligned}
$$

Les paramètres sont la raideur de la liaison, $k_{s}$ et sa longueur d'équilibre, $l_{0}$, la raideur de l'angle de liaison, $k_{\theta}$ et sa valeur d'équilibre, $\theta_{0}$. L'énergie liée aux déformations des angles de torsion est prise égale à

$$
E_{\omega}=\sum V_{n}(1+\cos n \omega)
$$

Le paramètre est cette fois la valeur de la barrière de potentiel associée à la torsion. L'entier $n$ traduit le caractère périodique du potentiel autour de la liaison centrale.

L'énergie d'interaction de Van der Waals et l'énergie d'interaction coulombienne interviennent entre des atomes non liés. Elles sont considérées respectivement de la forme :

$$
E_{v d w}=\sum \varepsilon\left[\left(\frac{r_{m}}{r}\right)^{12}-2\left(\frac{r_{m}}{r}\right)^{6}\right]
$$

pour l'énergie de Van der Waals, dans laquelle les paramètres sont la profondeur du puits de potentiel, $\varepsilon$ et sa position, $r_{m}$ et

$$
E_{c}=\sum \frac{q_{i} q_{j}}{D r_{i j}}
$$

pour l'énergie de Coulomb.

On désigne sous le nom de champ de force l'ensemble des expressions analytiques et des paramètres qui interviennent dans la description de l'énergie. Ces paramètres dérivent le plus souvent de données expérimentales. Nous avons donné la forme la plus courante de l'expression de l'énergie. Certains champs de force font apparaitre explicitement d'autres interactions.(telles que les liaisons hydrogène).

Lors de l'utilisation d'un champ de force, il convient de prendre quelques précautions :

i) Les champs de force sont paramétrisés pour des interactions particulières ou sont spécifiques à un ensemble précis de molécules. En général, il ne faut pas affiner les paramètres sauf si on dispose de suffisamment de données.

ii) Le terme de torsion pose souvent des problèmes parce qu'il provient à la fois des contributions internes et des contributions non-liées.

iii) Le terme répulsif en $r^{-12}$ dans l'équation Lennard-Jones des interactions Van der Waals n'est pas physiquement raisonnable aux faibles distances. Une forme exponentielle (le potentiel de Buckingham) qui s'écrit

$$
E_{v d w}=A \exp (-B r)-\frac{C}{r^{6}}
$$

est certainement meilleure mais son évaluation prend plus de temps.

iv) Les interactions de Van der Waals sont considérées comme isotropes et dans certains cas (les cas où les doublets libres ou les liaisons hydrogènes sont à prendre en compte), un traitement plus spécifique peut s'avérer nécessaire.

Plusieurs de ces problèmes sont résolus à l'aide du concept d'atome-type. Par exemple, un atome de carbone peut avoir une hybridation $s p, s p 2$ ou $s p 3$ ou être résonnant. Les 'donneurs' ou 'accepteurs' de liaison hydrogène peuvent être aussi définis dans certains champs de force. 
Le choix des charges qui interviennent dans l'expression de l'énergie coulombienne constitue certainement l'un des aspects les plus controversés de la modélisation, et les systèmes nécessitant un traitement précis des charges sont certainement à éviter par les débutants. Dans le cas le plus simple, des charges sont attribuées à chaque atome (ce sont les charges partielles) et l'énergie est calculée avec une certaine approximation concernant la constante diélectrique. Pour une molécule diatomique dont on connaît le moment dipolaire, l'attribution des charges est aisée, mais en réalité il n'existe pas de moyens non ambigu d'obtenir des charges à partir de la fonction d'onde.

La méthode la plus fiable consiste à calculer le potentiel électrostatique à partir de méthodes ab initio (développées plus loin), puis d'affiner les charges sur des points particuliers de la molécule (généralement les positions atomiques, parfois aussi les doublets libres d'un atome) de manière à reproduire le potentiel électrostatique. Pour de grandes molécules, cette méthode est appliquée à des fragments. Une rapide évaluation des charges peut aussi être effectuée empiriquement à partir de la nature des atomes de la molécule et leur connectivité. Ces paramètres utilisent les potentiels d'ionisation atomique et les affinités électroniques. Cette méthode est suffisamment rapide pour autoriser une modification de l'équilibre des charges durant une simulation de dynamique moléculaire.

\subsection{Les méthodes ab initio}

Pour de petits systèmes, il est possible de calculer la fonction d'onde à l'aide de certaines approximations, et d'obtenir ainsi toute l'information désirée. Les méthodes empiriques sont parfois paramétrées à partir des méthodes $a b$ initio. Et contrairement aux méthodes empiriques, les seules informations à fournir, en principe, sont les numéros atomiques et les positions atomiques. Il existe de nombreux articles traitant de ces méthodes. Nous n'en donnerons ici que les concepts de base.

Le but des méthodes ab initio est de résoudre l'équation de Schrödinger d'un système afin d'obtenir la fonction d'onde du système. Il est nécessaire pour cela d'effectuer de nombreuses approximations (méthode Hartree-Fock ou HF).

Les méthodes ab-initio utilisent l'approximation de Born et Oppenheimer, selon laquelle le comportement des électrons dans une molécule peut. s'étudier en supposant les noyaux fixes. On commence par construire les orbitales atomiques sous la forme d'un produit de fonctions d'onde monoélectroniques connues. Il ne s'agit pas en réalité d'un produit simple mais d'un déterminant:

$$
\psi=\chi_{1} \chi_{2} \chi_{3} \chi_{4} \ldots \ldots . \chi_{n}
$$

Pour les orbitales moléculaires, $\Psi$, on utilise une approximation analogue (déterminant de Slater)

$$
\Psi=\phi_{1} \phi_{2} \phi_{3} \phi_{4} \cdots \cdots . \phi_{n}
$$

où chaque fonction tridimensionnelle $\phi_{\mathrm{i}}$ contient les propriétés d'un seul électron de la molécule.

Chacune de ces fonctions précédentes $\phi_{i}$ s'écrit comme une combinaison linéaire d'orbitales atomiques connues (ou fonctions de base)

$$
\phi_{i}=\sum_{k} c_{i k} \chi_{k}
$$

Il reste alors à déterminer les coefficients $c_{i k}$ de ce développement.

Pour chaque électron, $i$, l'équation de Schrödinger est de la forme

$$
H \sum_{k} c_{i k} \chi_{k}=\varepsilon_{i} \sum_{k} c_{i k} \chi_{k}
$$

Après intégration sur tout l'espace, nous obtenons

$$
\sum_{k} c_{i k}\left(\int \chi_{l} H \chi_{k} d V-\varepsilon \int \chi_{l} \chi_{k} d V\right)=0
$$

Cet ensemble d'équations admet une solution non triviale si son déterminant est nul, soit 


$$
\operatorname{det}\left|H_{l k}-\varepsilon S_{l k}\right|=0
$$

où, par convention:

$$
\begin{aligned}
& H_{l k}=\int \chi_{l} H \chi_{k} d V \\
& S_{l k}=\int \chi_{l} \chi_{k} d V
\end{aligned}
$$

Le calcul de ces éléments de matrices nous permet de multiplier ce déterminant avec un polynôme en $\varepsilon$. Les solutions de cette nouvelle équation sont les énergies des orbitales. Ces énergies sont reliées aux équations séculaires pour obtenir les coefficients $c_{i k}$.

Deux problèmes principaux existent. Premièrement, $H$ dans l'équation monoélectronique comprend un grand nombre de termes. Deuxièmement, l'Hamiltonien lui-même contient différentes fonctions $\phi$ que nous cherchons à déterminer! Pour cela nous utilisons une approche dite 'auto-cohérente' (self consistent field SCF) où les valeurs initiales de $c_{i k}$ sont 'devinées'. Les solutions correspondent à des valeurs de $\varepsilon$ utilisées pour obtenir les meilleures valeurs de $c_{i k}$ jusqu'à ce que la convergence soit atteinte.

Il reste cependant un problème de taille - l'énergie de corrélation.
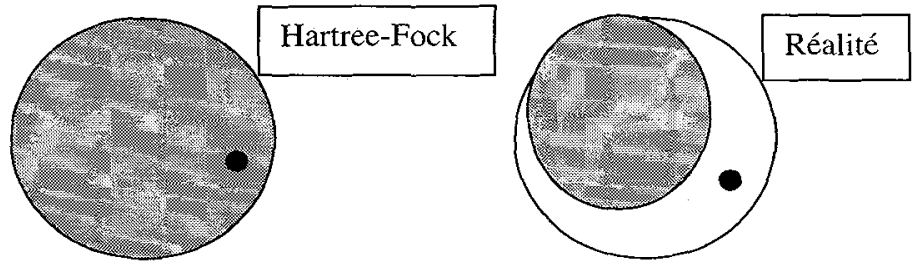

Figure 4 : Corrélation de deux électrons

Considérons deux électrons comme il est représenté sur la figure 4. Dans le traitement Hartree-Fock, le premier électron (disons $1 s$ ) est considéré comme moyenné sur une sphère avec laquelle le second électron interagit. En réalité, il y a une forte probabilité pour que les deux électrons soient de chaque coté du noyau. Parce que l'appariement des électrons change peu avec la conformation, les positions des noyaux dans les minima d'énergie sont généralement correctes, mais la forme du potentiel près de ces minima est fausse. Il en résulte que les géométries moléculaires sont prédites correctement, mais que les constantes de force et les énergies de dissociation nécessitent un traitement supplémentaire.

\subsection{Méthodes de Ia fonctionnelle de densité (Density Functional Theory DFT)}

En 1964, Walter Kohn a montré que l'énergie d'un système est totalement déterminée par sa densité électronique. Cette quantité est plus facilement manipulable que la fonction d'onde dans l'équation de Schrödinger. Tout ce dont nous avons besoin est un ensemble d'équations dont les solutions donnent la densité électronique du système, $\rho$, et son énergie, $E$. Cette dernière s'exprime comme

$$
E(\rho)=E_{K E}(\rho)+E_{C}(\rho)+E_{H}(\rho)+E_{X C}(\rho)
$$

Où $E_{K E}$ est l'énergie cinétique, $E_{C}$ est l'interaction électron-noyau, $E_{H}$ est l'énergie coulombienne électron-électron, et $E_{X C}$ contient le terme d'échange-corrélation. Il est important de noter que la corrélation électronique est incluse mais qu'elle est paramétrée. Une densité incorrecte donne simplement une énergie supérieure à la valeur réelle.

La quantité la plus importante de cette équation est le terme d'échange-corrélation car il est inconnu. Il existe différentes méthodes de DFT qui se distinguent par des expressions différentes de ce terme. 
L'exemple le plus simple est l'approximation de la densité locale (Local Density Approximation ou LDA) qui utilise la fonctionnelle locale d'un gaz électronique homogène.

Afin de simplifier le calcul, on suppose que seuls les électrons de valence déterminent les propriétés physiques du système. Des pseudopotentiels sont alors utilisés dans $E_{C}$ pour représenter le potentiel ionique de cœur. Il existe plusieurs expressions analytiques des pseudopotentiels. Ces derniers comportent des paramètres ajustés sur des données expérimentales

Les méthodes DFT sont généralement considérées comme des méthodes $a b$ initio, mais il y a toujours discussion pour savoir à partir de quel moment ou à partir de quelle 'quantité' de paramétrisation nous pouvons utiliser le terme $a b$ initio.

\subsection{Les méthodes semi-empiriques}

La matrice comprenant les éléments $H_{l k}$, de la forme (13a) est très coûteuse en temps de calcul et ce temps crôt à la puissance quatrième du nombre de fonctions dans la base. Les méthodes semi-empiriques s'appuient sur la méthode Hartree-Fock décrite dans les méthodes ab-initio (2.2). Mais cependant elles utilisent des approximations supplémentaires plus ou moins drastiques, selon lesquelles $H$ n'est pas précisément défini. En outre certains, voire tous, parmi les éléments $H_{l k}$ de la matrice $H$ sont remplacés par des paramètres. Il existe un nombre sans cesse croissant de sigles associés à des méthodes qui négligent le recouvrement des orbitales (neglect of overlap (NO)): INDO, MINDO, ZINDO, CNDO. Cependant, les paramétrisations les plus récentes, AM1 et PM3, peuvent donner des résultats tout à fait satisfaisants et sont considérablement plus rapides que les méthodes ab-initio.

\section{UTILISATION DES CALCULS D'ENERGIE:}

\subsection{Minimisation}

En pratique, il est plus courant de rechercher la structure ou la conformation correspondant au minimum de l'énergie du système dans le but de confirmer la validité de calculs que pour obtenir la géométrie du système elle-même. Par exemple, il est essentiel de s'assurer que le champ de force utilisé dans les simulations de dynamique moléculaire est capable de reproduire la structure mesurée, moyennée dans le temps, avec un degré de précision acceptable. Pour les molécules, il est courant de commencer avec des valeurs 'raisonnables' des longueurs de liaison, des angles etc... et d'utiliser ensuite un algorithme de minimisation parmi les méthodes choisies (champ de force, ab-initio...). Quant aux structures cristallines, elles peuvent, en principe, être prédites en minimisant l'énergie. Cependant la présence de plusieurs minima relatifs rend la tâche difficile, mais potentiellement 'rentable'.

\subsection{Dynamique}

La dynamique moléculaire simule le mouvement d'un système d'atomes en fonction des forces présentes. La force qui s'exerce sur un atome est simplement égale au gradient du potentiel effectif $V(r)$ qu'il subit. Elle peut s'évaluer pour chaque atome, à partir de petites variations $-\delta \mathrm{V}(\mathrm{r}) / \delta \mathrm{r}$. Connaissant à chaque instant la force qui s'exerce sur chaque atome, $i$, l'utilisation de la seconde loi de Newton permet de suivre l'évolution de'son déplacement, $s$, en fonction du temps: On obtient ainsi l'accélération

$$
a_{i}=\frac{\partial^{2} s_{i}}{\partial t^{2}}=\frac{F_{i}}{m_{i}}
$$

où $m_{l}$ est la masse de l'atome. L'intégration sur le temps conduit à la vitesse:

$$
\frac{\partial s_{i}}{\partial t}=\frac{F_{i}}{m_{i}} t+c
$$


A $t=0$ la constante est évidemment la vitesse initiale, $u_{i}$. La vitesse à tout temps $t$ est:

$$
\frac{\partial s_{i}}{\partial t}=a_{i} t+u_{i}
$$

Une intégration supplémentaire conduit au déplacement:

$$
s_{i}=\frac{1}{2} a_{i} t^{2}+u_{i} t+c
$$

où la constante est cette fois la position instantanée.

On pose comme hypothèse que l'accélération reste constante durant un pas de calcul, ce qui implique que nous devions faire des pas très courts (typiquement $10^{-15} \mathrm{~s}$ ). Pour l'étude de processus lents, ceci peut constituer une contrainte importante et plusieurs algorithmes ont été développés, le plus connu étant l'algorithme de Verlet.

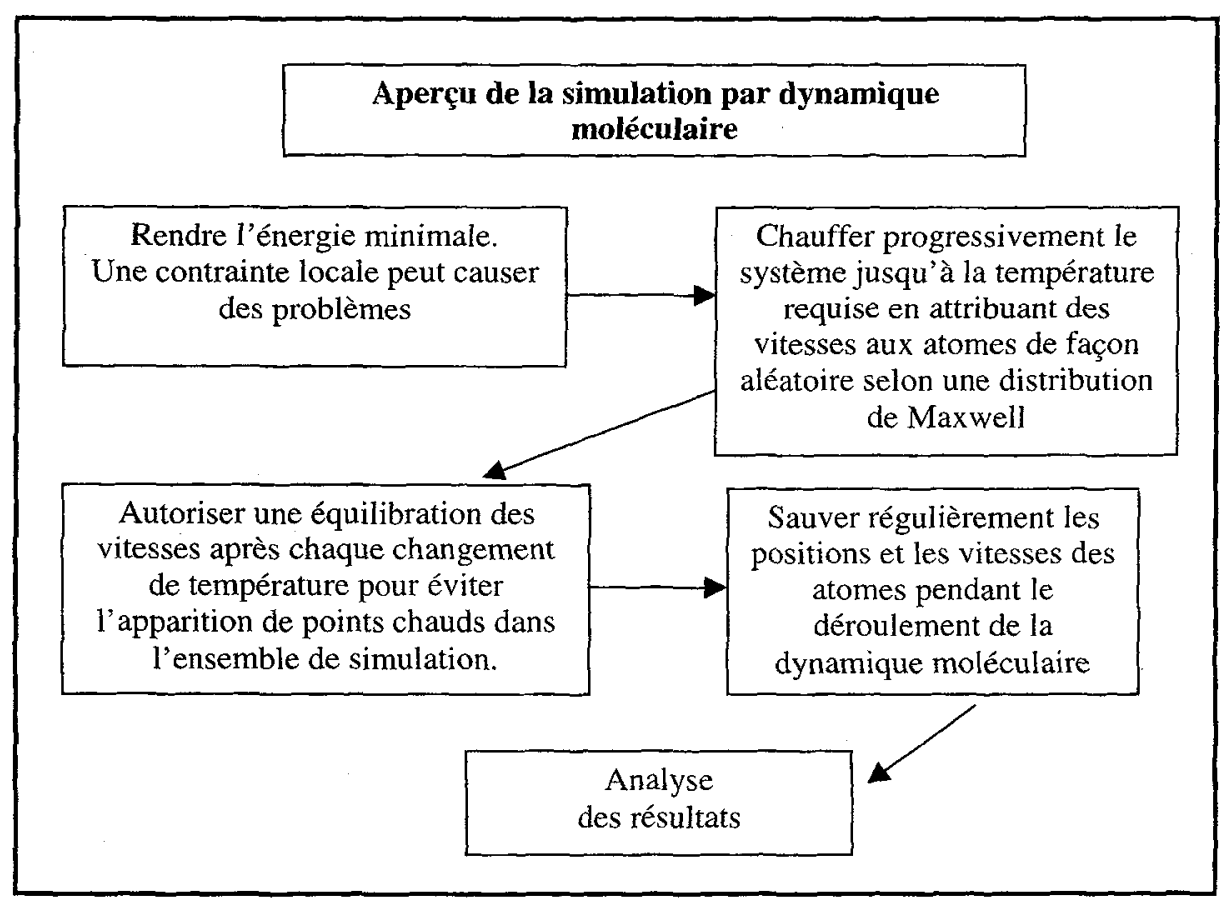

Figure 5 : Déroulement d'une simulation

\subsubsection{Analyse des trajectoires}

Les résultats les plus évidents auxquels conduisent les méthodes de simulation sont l'obtention d'une structure moyennée sur le temps et une image de la manière dont les distances et les angles varient en fonction du temps. (II faut noter qu'avec les simulations Monte-Carlo les propriétés dépendantes du 
temps ne peuvent pas être calculées.). La plupart des informations utiles à la diffusion neutronique proviennent des fonctions de corrélation du neutron dont la forme générale est:

$$
C_{A}(t)=\frac{\left\langle A_{i}(0) A_{i}(t)\right\rangle}{\left\langle A_{i}^{2}\right\rangle}
$$

Cette équation exprime la relation qui existe entre une propriété d'un atome (ou d'un groupe défini d'atomes) au temps $t=0$ et cette même propriété à un instant ultérieur $t$, moyennée sur toutes les molécules du système, et représentée en fonction de l'intervalle de temps. La durée nécessaire pour perdre la corrélation est le temps de relaxation ou temps de corrélation.

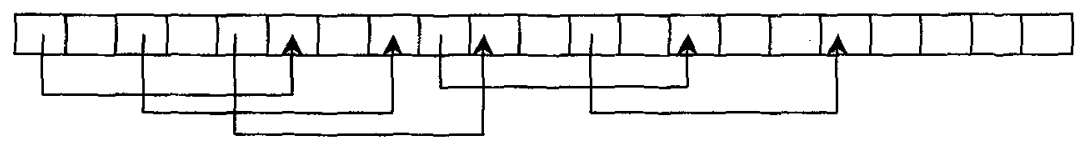

Figure 6 : Evaluation d'une fonction d'autocorrélation à partir d'une simulation

En supposant que la durée de la simulation soit nettement plus longue que le temps de corrélation, (ce qui est normalement le cas) plusieurs types d'informations peuvent être retirées de ce genre de calcul. Les énergies issues des simulations de dynamique moléculaire se doivent de donner une idée fídèle des barrières de potentiel réelles (dans la limitation imposée par le champ de force employé) parce que ces barrières seront traversées un certain nombre de fois et dans toutes les directions. De la même manière, si la simulation est suffisamment longue, il est possible d'en extraire la distribution des configurations et des conformations, et nous pouvons obtenir des valeurs moyennes afin de déterminer certaines quantités thermodynamiques. Cependant, souvent les changements aléatoires du système par une méthode Monte Carlo donnent un meilleur ensemble pour la thermodynamique parce que le système a moins tendance à être 'piégé' dans un puits de potentiel.

\subsubsection{Pourquoi la dynamique moléculaire est elle intéressante?}

Les trajectoires atomiques sont produites uniquement avec un modèle, et en principe, aucun paramètre n'est ajustable. La fréquence et le temps de simulation sont reliés au mouvement dans l'espace réel:

$$
I(Q, t)=\frac{1}{N}\left\langle\sum_{i, j} \exp \left(i Q \cdot r_{i}(t)\right) \exp \left(-i Q \cdot r_{j}(0)\right)\right\rangle
$$

où $I(Q, t)$ est la fonction de diffusion intermédiaire, $Q$ est le transfert de moment, $r_{i}(t)$ est la position de l'atome $i$ au temps $t, r_{j}(0)$ est la position de l'atome $j$ au temps 0 , et $<>$ désigne une moyenne d'ensemble. Pour la diffusion cohérente, la somme porte sur toutes les permutations de $i$ et $j$ mais pour la diffusion incohérente, seuls les termes $i=j$ sont pris en compte. Toute l'information sur la variation des distances interatomiqueś en fonction du temps est contenue dans le terme

$$
I(Q, t)=\frac{1}{N}\left\langle\sum_{i, j} \exp \left(i Q \cdot\left(r_{i}(t)-r_{j}(0)\right)\right\rangle\right.
$$

La diffusion inélastique incohérente permet de mesurer la transformée de Fourier de la fonction de diffusion intermédiaire, le facteur de structure dynamique: 


$$
S(Q, \omega)=\frac{1}{2 \pi} \int \cos (\omega t) \cdot I(Q, t) d t
$$

La propriété de la diffusion incohérente de ne concerner que des particules isolées simplifie grandement le problème: le neutron mesure la décroissance de la densité d'un seul atome en fonction du temps et de l'espace. De son côté, la diffusion cohérente permet de mesurer la décroissance des corrélations interatomiques en fonction du temps et de l'espace. Le lien le plus commun entre la dynamique moléculaire et l'expérience est la fonction d'autocorrélation des vitesses:

$$
\mathrm{VACF}=\left\langle v_{i}(t) \cdot v_{i}(0)\right\rangle
$$

Si la loi de diffusion intermédiaire peut être exprimée à l'aide de fonctions décrivant des processus harmoniques à un seul phonon, la transformée de Fourier de VACF (FACF) est comparable à la loi de diffusion mesurée.

$$
\mathrm{FACF} \propto \omega \sinh \left(\frac{\hbar \omega}{k T}\right) \frac{S_{i}(Q, \omega)}{Q^{2}}
$$

Cependant, si les fréquences issues de la fonction d'autocorrélation des vitesses VACF sont souvent comparables aux données expérimentales, ce n'est généralement pas le cas des intensités.

Le facteur de structure élastique incohérent (EISF) correspond à la limite au temps infini de la fonction de diffusion intermédiaire. Par conséquent, il est possible de faire apparâtre cette limite dans la fonction de diffusion intermédiaire incohérente en l'écrivant sous la forme d'une constante, l'EISF, à laquelle s'additionne une fonction qui décroît au cours du temps et finit par s'annuler à un temps infini:

$$
I(Q, t)=\operatorname{EISF}(Q)+I^{\prime}(Q, t)
$$

La transformée de Fourier de cette fonction est:

$$
S(Q, \omega)=\operatorname{EISF}(Q) \delta(\omega)+S^{\prime}(Q, \omega)
$$

L'EISF reflète la probabilité de trouver un atome à la même place au temps $t$ et au temps 0 . Ceci nous amène à considérer la fonction d'autocorrélation de Van Hove, $G_{s}(r, t)$, qui représente la probabilité de trouver un atome à la position $r$ au temps $t$ si ce même atome était en $\boldsymbol{r}_{\boldsymbol{\theta}}$ au temps 0 :

$$
G_{s}(r, t)=\left\langle\left(r-r_{0}(t)\right) \cdot r_{0}(t)\right\rangle
$$

L'EISF pour un atome isolé de longueur de diffusion $b$ (généralement un hydrogène $H$ ) peut s'écrire:

$$
\operatorname{EISF}(Q)=b^{2} \int G_{s}(\boldsymbol{r}, \infty) \cdot \exp (i Q \cdot r) d^{3} \boldsymbol{r}
$$

D'où:

$$
\operatorname{EISF}(\boldsymbol{Q})=\frac{1}{N} \sum_{i} b_{i}{ }^{2}\left\langle\exp \left(\left.i Q \cdot \boldsymbol{r}_{i}\right|^{2}\right\rangle\right.
$$

Il faut s'assurer que la simulation par dynamique moléculaire est suffisamment longue pour obtenir une complète description de l'espace des conformations, sinon les résultats seront évidemment faussés. 


\section{UTILISATION DES SURFACES D'ENERGIE POTENTIELLE}

Pour explorer un espace de conformations trop énergétique pour la dynamique moléculaire ou pour traiter des systèmes quantiques, nous calculons généralement l'énergie, ou les dérivées de l'énergie, pour des conformations que nous imposons nous-mêmes.

\subsection{Vibrations}

L'exemple le plus commun en est probablement celui des vibrations moléculaires. Elles sont mesurées en perte d'énergie (du neutron) et à basse température afin d'éviter les effets dus aux mouvements thermiques. Par conséquent, une dynamique moléculaire à la même température ne peuplerait pas les plus hauts niveaux d'énergie vibrationnelle; d'autres méthodes sont alors plus appropriées. Pratiquement tous les programmes basés sur les méthodes ab-initio, DFT et semi-empiriques offrent la possibilité d'obtenir les constantes de force et les fréquences vibrationnelles (dans le cadre de l'approximation harmonique). Ces calculs ne sont possibles que pour des molécules isolées ou de petits clusters ce qui fait que les interactions intermoléculaires sont ignorées. Or la plupart du temps, les vibrations moléculaires sont dominées par les interactions intramoléculaires. Dans le cas contraire, les constantes de forces dans la maille peuvent être obtenues par une méthode de différence finie. Cela implique de bouger chaque atome, un par un, dans le modèle, pour trouver les dérivées de l'énergie. Une fois obtenue la matrice des constantes de force, $\boldsymbol{F}, l^{\prime}$ inverse de la matrice de l'énergie cinétique, $\boldsymbol{G}$, est construite à partir des positions atomiques et des masses atomiques. Nous devons alors résoudre:

$$
\boldsymbol{H A}=\boldsymbol{A \lambda} \quad \text { où } \boldsymbol{G}^{\prime} \boldsymbol{F}^{\prime}=\boldsymbol{H}
$$

où $\boldsymbol{A}$ est la matrice des vecteurs propres et $\lambda$ sont les valeurs propres. Ces termes sont reliés, respectivement, aux déplacement atomiques et aux fréquences de vibrations. Nous pouvons ainsi calculer le spectre de diffusion inélastique des neutrons (inelastic neutron scattering ou INS). L'intensité d'une bande mesurée par INS pour un mode normal de fréquence $\omega_{s}$ (en perte d'énergie du neutron, suivant la convention adoptée en diffusion incohérente) est proportionnelle à :

$$
\sum_{i} n_{i} b_{i}^{2} Q^{2}\left\langle u_{i}^{2}\left(\omega_{s}\right)\right\rangle Z(\omega) \frac{\exp \left(-2 W_{i}\left(\omega_{s}\right)\right)}{\mu \omega_{s}}
$$

où $n_{i}$ est le nombre d'atomes de type $i, \mu$ est la masse réduite du système pour le mode à $\omega_{s}$, et $Z(\omega)$ est la densité d'états vibrationnelle. $2 W$ est le facteur de Debye Waller pour les modes externes. Il est à l'origine des ailes de phonons sur les côtés des pics inélastiques. En pratique, ces ailes sont utilisées pour déterminer ce facteur de Debye Waller.

Il est normal de comparer le spectre expérimental au spectre calculé, dans la mesure où cela nous fournit un excellent test sur la validité des calculs ab initio (ou sur d'autres méthodes). Normalement, les fréquences calculées sont en excellent accord avec l'expérience, et ce sont les intensités qui constituent le test réel. Si nécessaire, les constantes de force peuvent être ajustées afin d'améliorer l'accord entre les intensités observées et calculées des spectres INS. Si votre modèle reproduit à la fois la structure cristalline mesurée et le spectre INS, vous pouvez considérer qu'il est proche de la réalité.

\subsection{Effet tunnel}

La modélisation des systèmes quantiques a été démontré lors d'un travail récent effectué à l'ILL. Dans les molécules contenant un groupe $\mathrm{CH}_{3}$ ou $\mathrm{NH}_{3}$ faiblement gêné, l'effet tunnel rotationnel peut être observé à très basse température $(\sim 2 K)$. Les transitions entre les niveaux d'énergie peuvent être mesurées par diffusion inélastique des neutrons. Nous utilisons la structure cristalline, connue, comme point de départ de la modélisation. L'énergie du système est calculée en tournant le grouperment $\mathrm{CH}_{3}$ (ou $\mathrm{NH}_{3}$ ) pas à pas autour de son axe de rotation moléculaire. Cela nous permet d'obtenir le potentiel rotationnel, qui sera alors introduit dans un hamiltonien. Les valeurs propres de cet hamiltonien peuvent être calculées. Elles sont comparées aux transitions mesurées par INS. Cela nous permet de tester la validité des champs de 
force, celle des méthodes de calcul de charges ainsi que l'existence de couplages entre rotateurs ou de couplages du type rotation du groupement méthyle et translation de son centre de masse. Ce principe général (obtenir une fonction d'énergie potentielle et l'introduire dans un hamiltonien) peut s'appliquer à d'autres systèmes.

\section{CONCLUSION ; SI VOUS VOULEZ ESSAYER...}

La figure 6 est un résumé de ce que nous venons d'exposer. Si vous voulez vous lancer dans ces méthodes, les quelques conseils suivants peuvent vous être utiles :

-Choisissez un système simple avec une structure connue et une dynamique connue (avec des données inélastiques ou quasiélastiques existantes)

-Trouvez un programme commercial facile d'utilisation. Vous pourrez toujours utiliser le programme commercial ultérieurement.

-Introduisez la structure et choisissez le champ de force approprié. Minimise-t-il correctement la structure? Si oui, vous pouvez essayer la dynamique

-Jouez avec les options pour voir ce qui se passe. Le calcul est-il valable?

-Essayez 2 ou 3 systèmes de ce type avant de vous attaquer à un 'problème réel'

-Prenez conseil auprès des 'experts' aussi souvent que possible

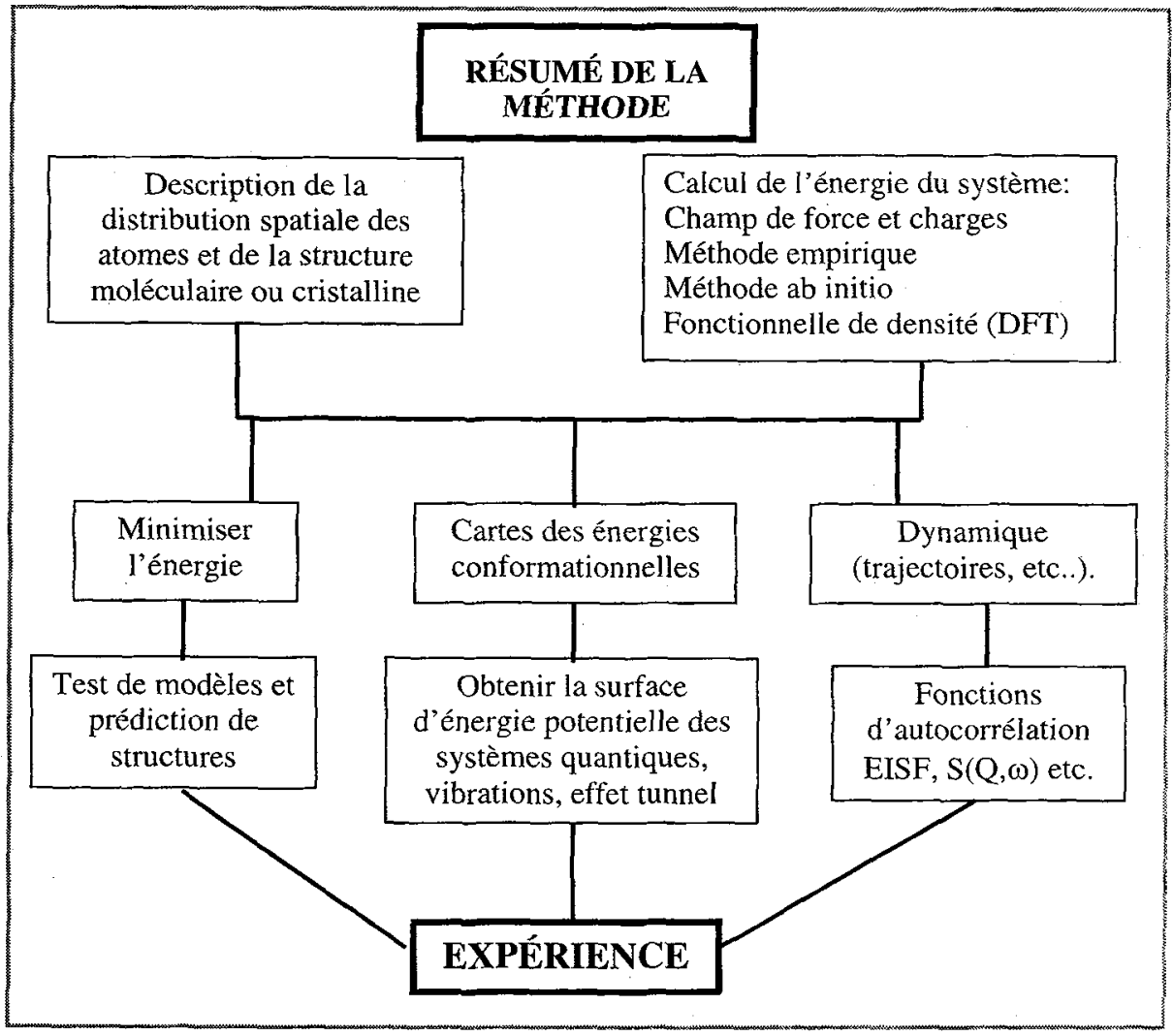

Figure 7 : Les possibilités offertes par les techniques de simulation et leur déroulement. 


\section{Références}

[1] Egelstaff P.A., Thermal Neutron Scattering (Academic, New York, 1971).

[2] Lovesey S.W., Theory of Neutron Scattering from Condensed Matter (Clarendon, Oxford, 1984).

[3) Squires G.L., Introduction to the Theory of Thermal Neutron Scattering (Cambridge University Press, Cambridge, 1978)

[4] Springer T., Quasielastic Neutron Scattering for the Investigation of Diffusive Motions in Solids and Liquids, Springer Tracts in Modern Physics (Springer, Berlin, 1972)

[5] Bée M., Quasielastic Neutron Scattering; Principles and Applications in Solid State Chemistry, Biology and Materials Science (Adam Hilger, Bristol, 1988) 